\title{
DEVELOPMENT AND PRACTICAL APPLICATION OF NEURAL INFORMATION TECHNOLOGIES, PATTERNS
}

\author{
Denis Gafurov ${ }^{1}$, Oleg Gafurov ${ }^{2, \mathrm{a}}$ \\ ${ }^{1}$ Krasnoyarsk Research Institute of Geology and Raw Material, 660049, Krasnoyarsk, Russia \\ ${ }^{2}$ International Laboratory of Vision Systems, Tomsk State University, 634050, Tomsk, Russia
}

\begin{abstract}
In this work, authors cover the basics of thinking feasibility of the acquired practical experience of application of methods, technologies of artificial neural networks application based on neuroemulator of neuromorphic computers with patterns formation. Study provides results of patterns formation in 2D and 3D formats in seismic wave fields of Verkhnechonsk field There is also forecasting based on pattern formation results on licensed sites that has led to petroleum discovery.
\end{abstract}

\section{Introduction}

The field of the computer sciences formed on complex of artificial intelligence, statistics and the database theory designated as Data Mining (detection of knowledge in data).This term means data recieving process from "crude" data, which allows getting new, potentially useful information about subject field. in this term along with.Except classical methods of the mathematical analysis and numerical modeling, this term includes the theory of neural networks.

Relevance of researches in this direction is confirmed by a lot of various applications of neural networks. Which are following:

- automation of image recognition processes;

- adaptive management;

- approximation of functionalities;

- forecasting;

- creation of expert systems;

- organization of tag memory and many other applications.

The approach focused on association of learning ability and interpretation principles corresponds to the purpose and tasks of Data Mining. The concept of templates (patterns) reflecting fragments of multidimensional relationship in data form modern technology of Data Mining (discovery - driven data mining).

These templates represent the regularities appropriate to subsamples of data, which can be compactly expressed in a form clear to the person. We carried out search of templates by methods, which are unbounded by framework of prior assumptions of sample structure and a type of values distributions of the analyzed indicators.

\footnotetext{
${ }^{a}$ Corresponding author: oleg.gafurov@mail.ru
} 
A lot of years the IBM corporation works together with DARPA on the creation of neuromorphic chips (Systems of Neuromorphic Adaptive Plastic Scalable Electronics, SyNAPSE), implementation of the project has begun in 2008. The purpose is creation of chips and systems, work of which will be organized by operation principle of neurons of animals' brain, for example, rodents.

Two stages - training and recognition, on the basis of an artificial neural network, are essentially different and each of them based on own software, partially integrated on a grade level.

In this work authors offer realization of pacquired practical experience of application of methods, technologies of artificial neural networks application based on neuroemulator of neuromorphic computers with patterns formation.

In present work we used empirical and theoretical methods of research, and work is based on already working intellectual systems implemented in production, created in different years with personally participation of the author under the leadership of O. M. Gafurov, D.O.Gafurov, V. I. Syryamkin and following intellectual neural information systems (INIS) "NeuroInformGeo", INIS "NeuroRobot", INIS "NeuroFinExpert", etc. Problem solving based on experimental data and the known theoretical provisions of adaptive management systems, neuroinformation technologies, "Data mining" methods, acoustics, machine vision and navigation.

Reliability of the received results is confirmed by a correctness of developed mathematical models, their adequacy on the known criteria for studied processes evaluation, by use of the known provisions of fundamental sciences, convergence of the received theoretical results with data of experiment and results of commercial operation of systems, and also results of researches of other authors. Reliability of novelty of a technical solution is confirmed by two patents for inventions and copyright certificates $[1,2]$.

Complexing of geologic-geophysical data was carried out by means of the mathematical apparatus of neural networks realized in "NeuroInformGeo" intellectual geographic information system, developed in "Informgeoservice" LLC (O. M. Gafurov) [3].

\section{Human brain}

Human brain is millions of years and has rather young formations. Human brain parts formations occurred at different times of evolutions and each of them independent selfregulating system. We allocate the main formations in a brain: cerebral cortex of brain, spinal bulb and diencephalon. The nervous centers which are responsible for hunger and saturations, located in diencephalon brain sites. Respiratory control apparatus pledged in spinal bulb.

\section{Thinking bases}

Neurophysiologists formulated the law of neuron impulses transmitting in synapses, identified visual and acoustical zones, created a map of projections the thalamic nidus on cerebral cortex of brain, etc.

General cognitive structure consists of following elements: visual, acoustical, tactile, somatic zones, all elements impose to live neural network (Figure 1). Calculation method based on trained neural networks which constantly existing during neurorobots creation or the birth of an animal or human. Existing neural networks form system of unconditioned reflexes, which have to provide survival rate of organism or neurorobots' live in real continuum (for the human is mother Earth of the Solar system in our Galaxy), in the same 
system additional skills are created, i.e. in other parts of a brain new neural networks (conditioned reflex) are created.

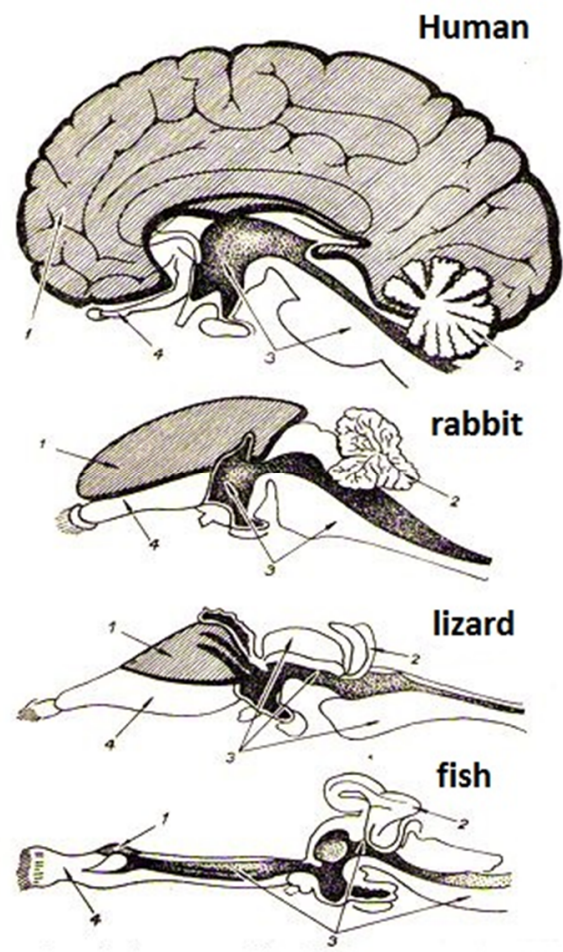

Figure 1. Development ofcerebral cortex of brain of fish, lizard, rabbit and human: 1 - cerebral cortex of brain; 2 - cerebellum; 3 - brainstem ; 4 - rhinencephalon.

The main paradigm in all calculations is the neural networks, already ready, congenital, transferable by inheritance or in our case, formed at the previous stage or the previous generations of neurorobots of neuronets library and trained neural networks during life of animal or human, and during functioning of the neurorobot in real time (patterns).

\subsection{The main concepts of brain working}

1. Congenital (unconditional) reflexes are received generally during evolutionary development of our body and the providing his binding to this planet to dayly surface and an opportunity to return to the Ocean.

2. Conditioned reflexes - a basis of training and retraining of neural networks.

3. Function of image recognition - fundamental property of wildlife. Essentially important feature of images recognition by neural network is ability of image restoration according to the reduced, incomplete or distorted data, stored in memory.

For example, the simplified image or part of image represented to recognition, a goal of a neural network is to restore the full key image which is stored in memory.

4. Modeling of external processes and events (the dream of the person is time of cleaning, at the same time continuous repetition of already taking place marvelous events, allocation of the most significant and their storing and retraining or fixing of already retrained networks). 
5. Connection of training and retraining, (in organisms it is usually connected on feedback with painful process) recognition and modeling of really taken place physical events and processes leads to emergence of conditioned reflexes and their fixing, i.e. emergence of skills.

6. Chatic dynamics of magnetic and electrical activity of a brain. Researches showed that electroencephalograms (EEG) of animals and the person, and also magnetic the fields generated in interneural tissues of a brain represent the determined chaotic processes with a small number of levels of freedom.

7. Parallel methods of the entering and modelled information processing in a brain.

8. Magnetic properties of cells of a glia (glial cells).

9. Subsections of neural network structure of a brain (subnet).

10. Oscillation of different parts of a brain, resonance and submission of processed signals again to the first signal system in visual, acoustical, somatic zones in complete or partial volume.

\subsection{Brain modeling and research projects}

\section{Europe: Human Brain Project}

One of the well known projects of brain modeling is Human Brain Project (HBP). A team from Swiss Federal Polytechnic School in Lausanne under the leadership of Professor Henry Markram.

In 2013 HBP won European Commission grant for 1 million euro. The Human Brain Project aims to combine all knowledge and investigation about brain to common model in supercomputer.

\section{USA: BRAIN Initiative}

This project calls as Brain Activity Map Project (BAMP). According to project name, there is a prediction: for 10 years, it will be possible to record and map activity of each neuron in human brain. Presidential Administration approved BAMP in 2013. It was planned to allocate 3 billion dollars from federal budget to project implementation.

\section{Human Connectome Project}

Human Connectome Project started in 2009 by National Institute of Health USA. As BAMP project, it is logical progeny of «Human genome». Human Connectome Project aims to map neurons connection in human brain more complete

\section{SyNAPSE}

Systems of Neuromorphic Adaptive Plastic Scalable Electronics SyNAPSE experiment stands apart of all called projects. SYNAPSE funded by DARPA and IBM. Project supervisor Dharmendra Modha aims to create non-virtual, real copy of brain, materialized as micro schemes with artificial neuron, fixed on silicone base [4].

\section{Neural networks}

Neural network methods are methods based on the use of different types of neural networks (NN). NN consists of elements called formal neurons, which imitate the work of neurons in the cerebral cortex. Each neuron transforms a set of signals coming to its input into an output signal. Figure 2 shows an example of formal neuron work. 


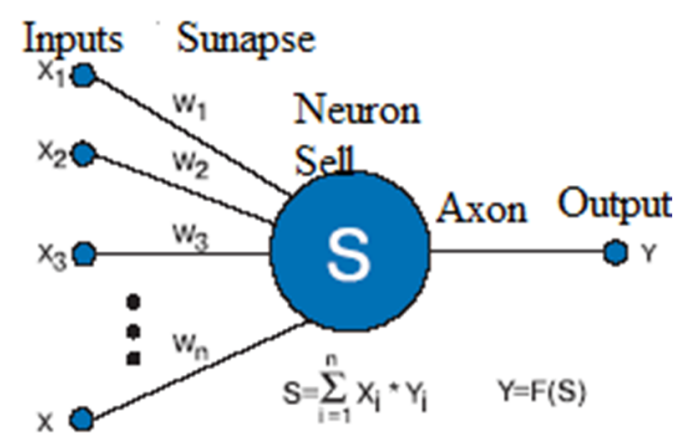

Figure 2. Formal neuron.

Figure 3 shows neural network structure.

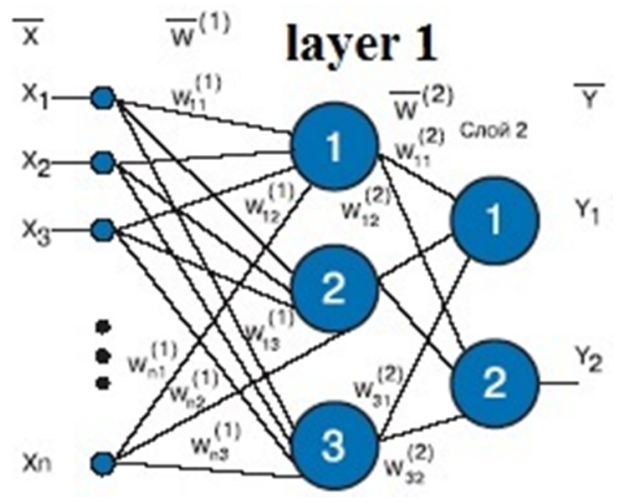

Figure 3. Neural network.

Connection can exist between individual neurons. Connections between neurons encoded by weight coefficients play a key role in function of the nervous system. On the Figure, connections between neurons are signed by Latin letter W. Index on the top means belonging of a weight coefficient to a layer. One of the advantages of the nervous system is the possibility of parallel operation of all the elements, which considerably increases the efficiency of problem solving in general. This feature of the nervous system is successfully used in image recognition systems. The neural network has inputs $\mathrm{X}$ and outputs $\mathrm{Y}$. It represents a system, which generates output state depending on the input state. Presence of weight coefficients that can be determined algorithmically, allows the nervous system to have the most important property - the ability to learn.

\subsection{Architecture of neural board and neuroprocessor}

Each neuroprocessor makes calculations for all assigned neurons, because the neural board implements sigmoid activation multi-layered perceptron. Each neuron calculates the output, as well as $\mathrm{y}=\operatorname{sigmoid}(\mathrm{P} \mathrm{i}(\mathrm{xi}-\mathrm{wi})$ ), where $x i$ is a contribution in neuron and $w i$ is corresponding weight. Figure 4 shows that bounded buffer keeps weight coefficients. 


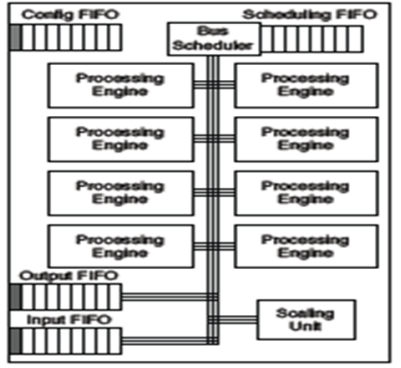

(a) An 8-PE NPU

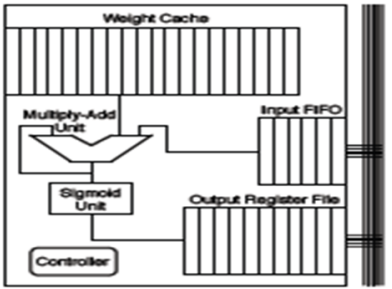

(b) A single processing engine

Figure 4. Scheme of neural board with 8 neuroprocessors and for one neuroprocessor (neuron).

Two stages - training and identification based on neural networks are different and each of them based on own software and partly connected on training stage. Diversification of networks types, training methods (with teacher, without teacher), techniques of weight coefficient calculations and problem solving for each case - makes difficult neurochips application in the process of basic study.

Combination of neuro-emulators and neurochips process operations is common principle of input attributes formation and running of weight coefficients, which were calculated by neuro-emulator in bounded buffer of neurochip.

\subsection{Neural network paradigm}

During image recognition, human unconsciously attracts a huge inventory of contextual knowledge, which is accumulating throughout all the life. Application of the neural network methods is able to simulate the processes happening during images recognition in human brain, which as first order can be presented in the form of a neural network of big complexity. One of the examples is neural network application method based on trained neural networks, implemented in "Neyrocyber" INS. Human don't operate by physical object, he operates by measured physical fields of this object.

As an example of neural networks application with patterns formation, we will consider in physical objects searching in seismic wave fields.

Physical fields, observed above oil-and-gas field, differentiate in dependence of diggings position and reconversation of rock mass affected by hydrocarbon migration. They allow creation of multidimensial attribute space, which can be used as actual basis in reservoir limits forecasting for tasks of complex interpretation.

Research of full volume ratio of geologic environment implemented by geophysical methods (seismic and magnetic measurements, etc.).Three-dimensional matrix is forming during the research of fields (magnetometry, gravimetry, etc.) or on the basis of constrain energized fields (seismic and electric measurements, etc.).

Computer sciences, which analyze digital image data, was formed on the combination of artificial intelligence, statistics, database theory and calls Data Mining. This term means data recieving process from "crude" data, which allows getting new, potentially useful information about subject field. in this term along with.Except classical methods of the mathematical analysis and numerical modeling, this term includes the theory of neural networks.

Approach oriented to training and interpretation principles combination fits to aims and tasks of Data Mining. At the heart of Data Mining (discovery-driven data mining) is conception of patterns, which show the fragments of multiple-aspect relations in data. Patterns are regularities of data subsamples, which can be presented in compact and human- 
understandable form. Patterns search goes by method, which is unbounded by aprior suggestions of sample structure and analyzed variables allocation.

NeuroInformGeo implemented technique of intellectual analysis and interpretation of geophysical parameters, based on neural networks methods, with ranking of input .characteristics by level of relevance, which can estimate informative contribution of every parameter for forecast accuracy. Futher, we implemented methods, based on trained neural networks, it is a complex analysis of forecasting rock mass and mineralization zones by means of geological data, fields data, GIS data and seismic measurements data in 2D, 3D formats. User gets forecasting mechanism results in understandable form.

We can make experience accumulation of forecasting and interpretation in the type of formation, saving and correct usage of patterns, which is a library of neural networks trained on model fields or in drilled areas.

Forecasting accuracy on every stage provided by correctly defined parameters new drilled wells as truth information, which has absolute accuracy level for forecasting in geophysical fields. Such knowledge updates and forecasting of new wells drilling efficiency has to be developed by modeling and recalculation of new zones, based on previous experience accumulation, and by estimation of economic efficiency

Usually, people solve the task, which was described above, by means of classic methods of interpretation, by numerical modeling and statistical analysis.

\section{Pattern formation in intellectual neural informational system «NeurolnformGeo»}

\subsection{Geological bodies and their images in geophysical and geochemical fields}

We investigated all volume part of a geologic environment by geophysical methods (seismic and magnetic measurements, etc.). Three-dimensional matrix forms on the basis of forcibly excited fields (seismic and electric measurements) or during the research of natural fields (a magnetic and gravitation measurements, etc.). In matrix, real geological bodies and objects were replaced by digital images of their physical fields.

We inroduce a concept of physical model of the environment in the form of Qn $(x, y, z)$ function, which describes distribution of physical parameter in the area $B$. This parameter forms geophysical field $\mathrm{fn}(\mathrm{x}, \mathrm{y}, \mathrm{z})$ in surrounding space and has parameters of geological environment $p i, p k$ c. Following equation $\mathrm{fn}(\mathrm{x}, \mathrm{y}, \mathrm{z})=\mathrm{f}(\mathrm{Qn}, \mathrm{Pi}, . ., \mathrm{Pk})$ connects $\mathrm{Qn}$ и $\mathrm{f}$, in other words, formula allows calculating the field of geophysical parameter (one of the aims of geophysics).

To calculate the field of geophysical parameter fn $(x, y, z)$ we need a values of Qn $(x, y$, $\mathrm{z}$ ), values of geological environment parameters pi, pk and data about position of boundary (contact) surfaces. Density values of rocks - for seismic measurement, values of sandstones, seams of clay - for geological bodies and objects. In the field of measured wave fields, which were received as a reaction of real environment for forced excitation by source (explosion, vibration source, pulsed source, etc. ), boundaries counts as rapid change of oscillation amplitude. Fluctuation of density values creates increasing or decreasing of compressional velocity.

Further, set of geophysical and geochemical parameters shows secondary alteration of environment parameters affected by hydrocarbons or other mobile aggressive mediums.

Present scheme is simple and works for simple geological fields. However, all oil gas fields in the world have reference horizons - geological bodies, well known and extending on all area, with known characteristics (Southwest Siberia is a horizon U1). We can 
research more lighter effects in seismic fields but not always research of effects as geological body or object are not succeeded on short notice.

Creation of Qn (x, y, z) geological model creation direct task by fn (x, y, z) measured geophysical parameter calls inverse task. It is necessary to find distribution of $\mathrm{p} 1 \ldots \mathrm{pk}$ geological environment parameters and position of contact surfaces.

\subsection{General scheme of direct and inverse task solving}

Solving of direct task is $f$ fields calculation of $Q$ parameter dispersion by assign geological model, which consist of geological bodies, objects and their physical characteristics.

$$
\mathrm{Q}(\mathrm{x}, \mathrm{y}, \mathrm{z}) \stackrel{\mathrm{t}(\mathrm{Q})}{\longrightarrow} \mathrm{fQ}
$$

Inverse task is calculation and creation of model by measured field $\mathrm{J}(\mathrm{f})$ :

$\mathrm{J}(\mathrm{f})$

$$
\mathrm{fQ} \longrightarrow \mathrm{Q}(\mathrm{x}, \mathrm{y}, \mathrm{z})
$$

Usually every human or animal in everyday life operates with images of objects. For example, geophysicists operate with visual data - seismic measurements, smell geochemistry, etc.). Therefore we solve inverse task constantly, as human operates seen and heard information every minute.

We checked accuracy of geological model creation by means of direct task solving, because creation is a complex process. Mechanism of direct task solving is following: we calculate direct task by created geological model on basis of known function $Q n k 1$ and field of parameter $f 1(Q n)$. Then we compare calculated value $f 4(\mathrm{Qn})$ and measured value $\mathrm{f}$ (Q) of field. Taking into account difference and convergence properties of values, we change solving parameters of inverse task and specify geological bodies boundary or physical parameter distribution. This process runs until $U(Q)=\min [f 1(Q n)-f(Q)]$ function will be minimized.

We consider the next finite automat, which consists of five objects [A, S, Z, v, Q], where $\mathrm{A}=\{\mathrm{a} 0, \mathrm{a} 1 \ldots, \mathrm{an}\}-$ is finite input parameter list (measured geophysical fields) (Figure 5).

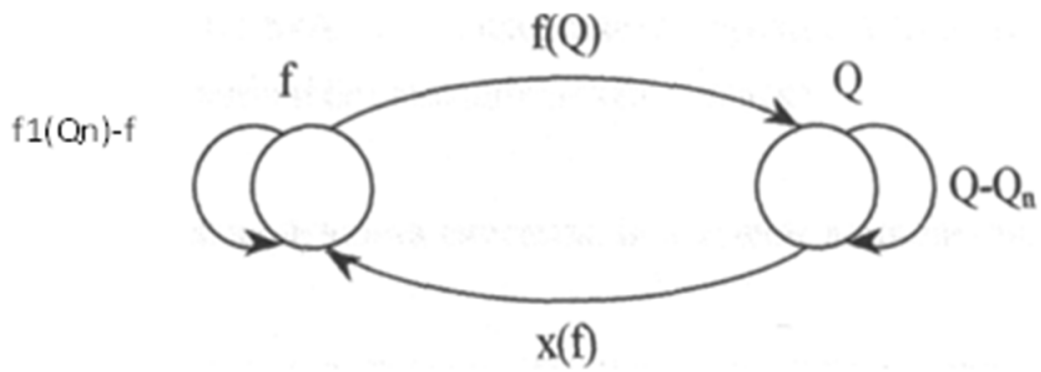

Figure 5. Finite automat, solving direct and inverse tasks.

$\mathrm{Z}=(\mathrm{Zo}, \mathrm{Zi}, \ldots, \mathrm{Zm}\}$ - output parameter list (geological model).

$\mathrm{S}=\{\mathrm{So}, \mathrm{Si}, \ldots, \mathrm{Sr}\}-$ set of internal states (processing function).

$\mathrm{v}: \mathrm{SxA} \rightarrow \mathrm{S}$ - state transition function (to the next state). 
$\xi: \mathrm{SxA} \rightarrow Z-$ output function (convergence estimation of calculated and measured fields of parameters).

Every time calculation of finite automat transition to the next state demands calculation of state transition function $\mathrm{v}: \mathrm{SxA} \rightarrow \mathrm{S}$ i.e. from one previous state it needs to transit to another. It might to correspond to define conditions of finite automat and transition needs introduction of control parameters for each step of determined process and needs to choose to which acceptable point it might to come.

If we consider this process by determined prototype of information processing in geophysics then it is a batch mode of data processing, in which bath mode makes the operating mode of geophysical data procedures operation. To train machine to solve tasks correctly on each step we need to introduce machine training term (or selftraining) after every processing of materials.

During the function, transition process we need to make decision based on implicit and unevident dependencies, which were coverted in aprior data and in data based on previous calculations because to control calculation process we need to make accurate tasks of transition function and control parameters as a concrete numbers. In this stage, in consider of set, which has or calculates dependences data by training of neural network, based on wells analysis data, correlation dependences of geological environment parameters, we form next transition. In other words, we define v: $\mathrm{SxA} \rightarrow \mathrm{S}$ state transition function and control parameters without human intervention (Figure 5).

\subsection{Principles of training of system, step type knowledge supplementation}

For common solution of connected, non-controversial geological model creation by its image in geophysical, geochemical fields, we created graph based on processing and interpretation functions. Today in controls by human on each stage of introduction of geophysical task control parameters. In other words, expert forms control U: S x A $\rightarrow$ S function.

Assume, that parameters formation of each function is propositions $\mathrm{P}(1), \mathrm{P}(2), \mathrm{P}(3), \ldots$, each of which might be either true or false. Principle of finite induction maintains that, to prove truth of $P(n)$ propositions for all $n 0 P$ we should to determine proposition of $\mathrm{P}(1)$ and infinite sequence of implications:

$$
\mathrm{P}(1) \Rightarrow \mathrm{P}(2)=>\mathrm{P}(3) \Rightarrow \ldots \mathrm{P}(\mathrm{n})=>\mathrm{P}(\mathrm{n}+1) \Rightarrow \ldots
$$

On each stage machine has to calculate parameters correctly and problem description in the beginning has to fit to real geological environment (Figure 6).

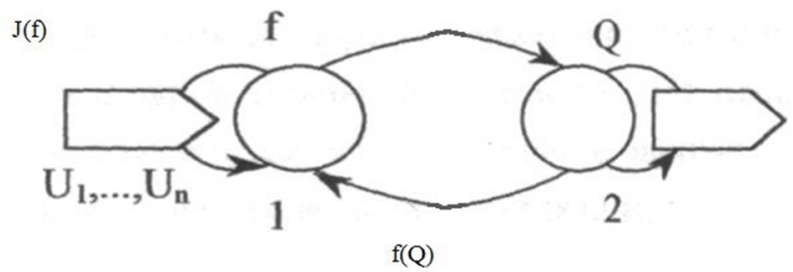

Figure 6. Finite automat, solving direct and inverse tasks of geophysics by means of neural computer technologies.

Neural computer program calculates parameters on each cycle, based on training set. 


\section{Pattern definition}

We can make experience accumulation of forecasting and interpretation in the type of formation, saving and correct usage of patterns, which is a library of neural networks trained on model fields or in drilled areas.

Correctly defined parameters of new drilled wells provides accuracy of forecasting on each step as reference information, which has absolute degree of accuracy for geophysical fields forecasting. Without subjective factor as opinion of geophysicist, this kind of step type knowledge supplementation and forecasting of new wells drilling efficiency, we need to add their modeling and recalculation of new zones, based on previous experience and estimation of economical efficiency of wells location points.

Physical model creation divided into two main stages - patterns formation as trained neural networks on sample objects and identification of research objects (Figure 7).

We check accuracy by regression equation, created by predicted and true values in wells.

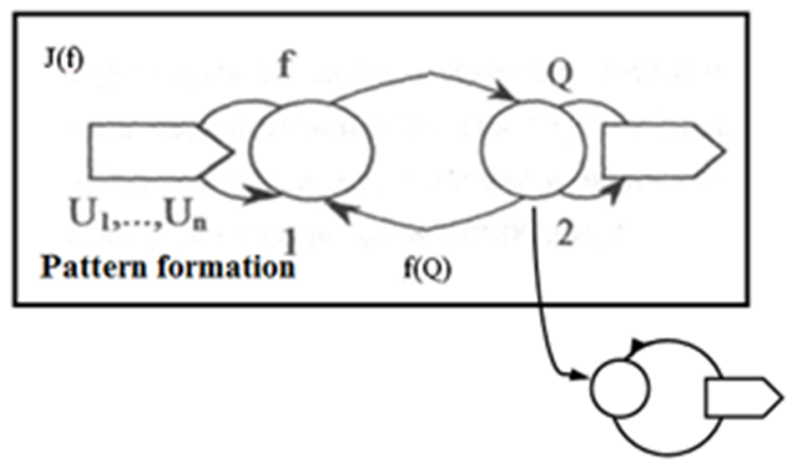

Figure 7. Patterns formation.

At the stage of data preparation, for sample (neural) networks formation and forecasting of advanced reservoir characteristics in Osa, Preobrazhensky, Ust-Kutsky horizons of Verkhneichersk and West-Chonsk license blocks we carried out following procedures. We implemented amplitude processing of seismic material, which includes AVO calculations, acoustic impedance calculations, trend model creation, calculation of scattered wave energy, attributes calculations, results of interpretation and productive horizons wells testing reports were presented.

At the stage of trained neural networks (patterns) formation, we prepared 22 wells for oil field and geological parameters forecasting in Nepsk high. Another 22 wells were prepared for perviousness of Verkhneichersk license block by 3D dram of Verkhnechonsk well bore. For 40 wells we established linear correlation with influx value in wells, forecasting of the effective thickness collertor and effective porosity $\left[\phi_{\mathrm{e}}\right]$ was explained and forecasting maps are created.

We formed and trained neural networks for forecasting of following parameters:

- net pay of zones ;

- porosity coefficient $\left[\phi_{e}\right]$;

- fluid content (absence, water, oil, gas);

- effective thickness collertor;

for 40 wells by geological parameters and 45 wells by influx, appropriated to Irkutsk license block. 
In result, we created forecasting maps for this parameters by using of all possible information about wells with GIS data interpretation by common complex of petrophysical dependences ("Shelf -South" LLC). Further, wells testing reports were presented ("RNKrasnoyarskpetroleum R\&D Institute" LLC), forecasting of Nepsk series was implemented based on 3D sample creation on Nepsk high.

According to the research performed we got geological geophysical model in INIS "NeuroInformGeo" for Verkhneichersk license block and trained neural network, which keeps coefficients of connections between geological, oil field parameters and attributes of measured geophysical fields.

Presented model contains numeric data and analysis instrument, which allows retraining of neural networks and building of new geological geophysical models on the basis of new data or modeling of constructing wells, taking into account models, created in previous stage.

Application of neurochips allows transition of counting process to the field, for example, flying machine equipped by instruments for magnetic and geochemical measurements in real time mode might to define indicators of mineral deposit fields.

TrueNorth is a brain-inspired chip architecture built from an interconnected network of lightweight neurosynaptic cores (Figure 8) [5, 6]. TrueNorth implements "gray matter" short-range connections with an intra-core crossbar memory and "white matter" long-range connections through an inter-core spike-based message-passing network. TrueNorth is fully programmable in terms of both the "physiology" and "anatomy" of the chip, that is, neuron parameters, synaptic crossbar, and inter-core neuron-axon connectivity allow for a wide range of structures, dynamics, and behaviors. Inset:The TrueNorth neurosynaptic core has 256 axons, a 256256 synapse crossbar, and 256 neurons. Information flows from axons to neurons gated by binary synapses, where each axon fans out, in parallel, to all neurons thus achieving a 256-fold reduction in communication volume compared to a point-to-point approach.

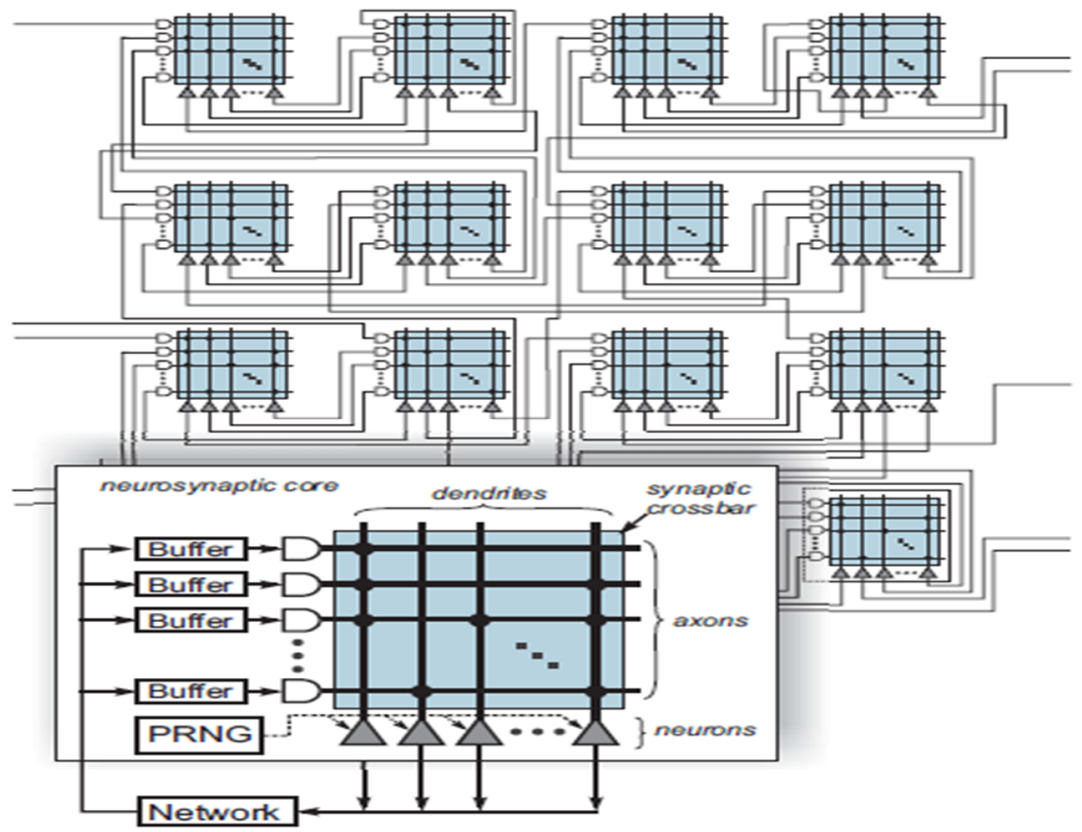

Figure 8. TrueNorth system. 


\section{Conclusion}

Development and practical application of neural information technologies have a new impulse by application of neuromorphic chips and their combination with neuroemulators, implemented in computer with traditional architecture. Firstly, research, calculations, pattern formation allow to emphasize set of tasks, for solving which we use neuroemulator and in future to solve identification tasks by trained neural network or neurochip.

IBM specialists makes interesting analogy on human brain example. They compare usual computer systems with left hemisphere. Neuromorphic systems might to find discrete patterns in big massive data, and interpret this information as right hemisphere. Perhaps, in future scientists will combine traditional computer possibilities and neuromorphic chips by creation of common super effective structure.

IBM, inspired by human brain, created new chip with architecture of computer, which consists of one million neurons and 256 million synapses. It is the biggest chip, which IBM ever created, it has 5.4 billion transistors and 4,096 neurosynaptic cores on chip. It consumes $70 \mathrm{~mW}$ during the real time operation - less than traditional chip. As a part of full cognitive ecosystem of hardware and software, this technology opens new calculation scopes for distributed sensory device and super calculating possibilities [7].

\section{Acknowledgment}

The paper was written as part of the research project No. 8.2.31.2015, carried out with the support of the Program "Research Foundation of Tomsk State University named after D.I. Mendeleev" in 2015 - 2016, grant RFBR No. 16-29-04388/16.

\section{References}

[1] O. M. Gafurov, D. O. Gafurov, M. V. Pankov, N. B. Krasilnikova, A. O. Gafurov, A. K. Bitner, A method of operational wells deposit places determination in case of hydrocarbons fields development Patent Russian Federation, MPK G01V1/00 (2006.01), G01V11/00 (2006.01)

[2] V.I. Syrijamkin, et al., Method of controlling moving object and apparatus for realizing said method Patent Russian Federation, MPK G01C21/14

[3] D.O. Gafurov, O. M. Gafurov, V.A. Kontorovich, Technology of seismic exploration (4), 85 (2014)

[4] Brain Inside the Computer: Projects of Neuromorphic Modelling. Naked Science \#26, August -September URL: https://naked-science.ru/article/nakedscience/braininside-the-computer-neuromorphic-modeling-projects

[5] P. Merolla et al., Proceedings of the Custom Integrated Circuits Conference, 6055294 (2011) doi: 10.1109/CICC.2011.6055294

[6] R. Preissl et al., International Conference for High Performance Computing, Networking, Storage and Analysis, 6468524 (2012) doi: 10.1109/SC.2012.34

[7] Brain Power. IBM. URL: http://www.research.ibm.com/cognitivecomputing/neurosynaptic-chips.shtml\#fbid=OMA2Lz7L_n4 